\title{
Effect of Bearing Type on Taper Material Loss in Hips from One Manufacturer
}

Harry Hothi ${ }^{1}$, Antti Eskelinen ${ }^{2}$, Johann Henckel $^{1}$, Young-Min Kwon ${ }^{3}$, Gordon Blunn ${ }^{1}$, John Skinner ${ }^{1}$, Alister Hart ${ }^{1}$

1. Institute of Orthopaedics and Musculoskeletal Science, University College London and the Royal National Orthopaedic Hospital, Stanmore, United Kingdom

2. The Coxa Hospital for Joint Replacement, Tampere, Finland

3. Massachusetts General Hospital/ Harvard Medical School, Boston, USA

Corresponding Author:

Harry S. Hothi

Institute of Orthopaedics and Musculoskeletal Science (University College London)

Royal National Orthopaedic Hospital

Brockley Hill

Stanmore, Middlesex

HA7 4LP

United Kingdom

Phone: +44 (0) 2089095825

Fax: $\quad+44(0) 2089548560$

Email: h.hothi@ucl.ac.uk 


\begin{abstract}
Background: Numerous studies have reported on clinical significant volumes of material loss and corrosion at the head-stem junction of metal-on-metal (MOM) hips; less is understood about metal-on-polyethylene (MOP) hips. We compared the effect of bearing type (MOM vs MOP) on taper material loss for a hip system of a single design.
\end{abstract}

Methods: In this cohort study, we recruited retrieved MOM $(n=30)$ and MOP $(n=22)$ bearing hips that were consecutively received at our centre.

We prospectively collected associated clinical and imaging data. We measured the severity of corrosion and volumes of material loss at each head taper surface and used multivariate statistical analysis to investigate differences between the two bearing types.

Results: The median rate of material loss for the MOM and MOP groups was $0.81 \mathrm{~mm}^{3} /$ year $(0.01-3.45)$ and $0.03 \mathrm{~mm}^{3} /$ year $(0-1.07)$ respectively $(\mathrm{p}<0.001) .29$ out of $30 \mathrm{MOM}$ hips were revised for adverse metal reactions, compared with 1 out of 22 MOP hips.

Conclusion: MOP hips lost significantly less material from their taper junctions than MOM hips.

Our results can reassure patients with MOP Pinnacle hips that they are unlikely to experience clinically significant problems related to material loss from the taper junction.

Keywords: Hip; Taper; Metal-on-polyethylene; Corrosion; Wear 


\section{Introduction}

Adverse reaction to metal debris (ARMD) is a common reason for revision of metal-on-metal (MOM) total hip replacements (THRs) [1]. The source of this debris is a combination of material lost from the cup-head bearing surface and the head-stem taper junction. Numerous studies have speculated that metal release (specifically cobalt and chromium) from the head taper surface, plays a large role in early failure [2-5]; indeed, clinical studies have shown that some resurfacing hips have a lower revision risk compared to hips with this junction [6].

More recently, several clinical studies have reported complications due to ARMD in metal-onpolyethylene (MOP) bearing THRs, with the source of metal debris attributed to the head-stem junction [7-8]. The Pinnacle (DePuy) THR cup is one of the most commonly implanted worldwide and has most often been paired with a MOP bearing [1]. The metal-on-metal (MOM) combination of this design was discontinued by the manufacturer in 2012 based on low market demand. In 2016, the National Joint Registry (NJR) of England, Wales and Northern Ireland reported a 10-year revision risk of 14.59\% for the MOM Corail-Pinnacle [1]. It is speculated that material loss from the taper junction is a contributor to some of these early revisions.

Retrieval centres analysing MOM Pinnacles revised due to ARMD, have reported volumes of material loss as high as $23 \mathrm{~mm}^{3}$ from the taper surfaces [9-11]; this is in contrast to $1.45 \mathrm{~mm}^{3}$ of metal loss which has previously been shown to be clinically insignificant [12]. It is unclear however whether surgeons and patients should expect to see the same scale of taper problems in the MOP hips.

In this study we collected Pinnacle hips with MOM and MOP bearings. We (1) analysed the taper surfaces of each component and (2) used multivariate statistical analysis to evaluate the scale of the differences in taper corrosion and material loss between the two groups. 


\section{Methods}

In this cohort study we included all consecutively retrieved Pinnacle hips, received by our laboratory during a 24-month period, that had MOP bearings and 12/14 head-stem junctions $(n=22)$. The bearings had been paired with either a Corail $(n=16)$ or Summit $(n=11)$ femoral stem, both made of the same titanium alloy. We also included the first thirty consecutively retrieved MOM bearing Pinnacle hips received at our laboratory. The bearings had been paired with $12 / 14$ stems consisting of the Corail $(n=11)$ and Summit $(n=19)$. The metal heads in both groups were composed of the same cobalt-chromium alloy.

We prospectively collected preoperative, intraoperative, and postoperative data concerning the revised arthroplasty.

\section{Visual Assessment of Head Taper Corrosion:}

The taper surfaces of all heads were examined macroscopically and with the aid of a Leica M50 microscope [Leica Microsystems, Germany] at up to 40x magnification to assess the presence and severity of corrosion. Each taper was graded with a score of between 1 (no corrosion) and 4 (severe corrosion) using the scoring system defined by Goldberg et al. [2]; this is known to be a reliable visual method which has shown to be correlated with measured volumetric material loss at the taper [3]. Higher corrosion scores were graded based on evidence of greater black debris, discolouration, pitting or etching on the surface.

\section{Measurement of Head Taper Material Loss:}

The volume of material loss at each of the head taper surfaces was measured using a Talyrond 365 (Taylor Hobson, Leicester, UK) roundness-measuring machine, using methods previously published [13]. A series of 180 vertical traces were taken along the axis of the taper surface 
using a $5 \mu \mathrm{m}$ diamond stylus. These were combined to form a rectangular surface from which unworn regions were identified and the volume of material loss in worn regions calculated.

\section{Detailed Visual Inspection}

Detailed macroscopic and microscopic inspection of the bearing surfaces was performed using a previously published method [14]. All inspections were performed by a single examiner experienced in retrieval analysis. The presence and severity of damage features of scratching (light, moderate, heavy), discolouration, pitting, presence of visible wear scars and any other notable surface changes were recorded.

In cases where the shell and liner components were retrieved and separated, we examined this junction for evidence of corrosion of the metal components or damage of the polyethylene components.

\section{Statistical Analysis:}

An initial set of analyses compared the characteristics of the two groups: (1) head size, (2) gender, (3) age, (4) time to revision, (5) inclination, (7) horizontal femoral offset and (7) vertical femoral offset. Categorical variables were compared between groups using Fisher's exact test. All continuous variables were found to be approximately normally distributed, and were compared between groups using the unpaired t-test.

Next the two outcome measures were compared between groups using a regression approach. A first analysis compared the groups without adjusting for any potentially confounding variables. The analysis was repeated, comparing groups after adjusting for factors found to show any difference between groups from the initial analyses (factors with a p-value of $\leq 0.2$ ). Taper wear rate was a continuous variable, and was thus analysed using linear regression. An examination of the distribution of the values suggested it was highly positively skewed. Thus, 
the variable was analysed on the log scale. A small constant was added onto all values before the transformation to enable the transformation to be applied to those with a zero wear rate. The Goldberg score took values from 1-4. The four outcome values were distinct categories, and cannot be considered as a continuous score. Due to the ordinal nature of the outcome, the analysis was performed using ordinal logistic regression.

\section{Source of Funding}

This independent study was funded by DePuy Synthes.

We confirm that all investigations were conducted in conformity with ethical principles of research, that informed consent for participation in the study was obtained and that institutional approval of the human protocol for this investigation was obtained.

\section{Results}

The two groups, MOM and MOP, were similar except for head diameter and time to revision (Table 1): 12 out of 22 MOP hips had 36mm heads whereas all MOM hips had 36mm heads $(\mathrm{p}<0.001)$; and the mean time to revision was 54 months for the MOP hips and 91 months for the MOM hips $(\mathrm{p}=0.005)$.

The MOM implants were retrieved from 13 female and 17 male patients, whilst the MOPs were retrieved from 12 female and 10 male patients. The median age of patients in the MOM and MOP groups was 63.7 years (48.8-75.3) and $63.1(36.0-80.4)$ respectively.

The median cup inclination angles for the MOM and MOP groups were $45^{\circ}(33-61)$ and $46^{\circ}$ (32-58) respectively; 7 of the MOM and 4 of the MOP cups were positioned outside of the Lewinnek safe zone, Figure 1. 
The median horizontal femoral offset of the implants in the MOM and MOP groups were $48 \mathrm{~mm}$ (30-55) and 44.5mm (28-57) respectively. The vertical femoral offset for the two groups was measured as 79.5mm (57-93) and 77.5mm (62-98) respectively, Figure 2.

\section{Reason for revision}

29 of the MOM hips were revised due to ARMD; 1 was revised for infection. The reasons for revision of the MOPs were cup loosening $(n=11)$, infection $(n=5)$, pain $(n=2), \operatorname{ARMD~}(n=1)$, recurrent dislocations $(n=2)$ and a periprosthetic fracture of the proximal femur $(n=1)$.

\section{Head-Stem Taper Corrosion}

A summary of the corrosion scores in the two groups are given in the Table 2 . The figures are the number and percentage of patients with each score in each of the two groups. Ordinal logistic regression was used to compare the scores between groups, Table 3 . The sizes of the group differences are expressed as odds ratios. This give the odds of being in the next highest score category (e.g. score 3 relative to score 2) for the MOP group relative to the MOM group. The results suggested a highly significant difference between groups both before and after adjusting for potentially confounding variables, with significantly lower scores in the MOP group, Figure 3. After adjusting for possible confounders, the odds of being in the next highest category were only 0.05 times as large (or 20 times lower) in the MOP group than in the MOM group. Typical examples of the taper damage of the two groups are presented in Figure 4.

\section{Measurement of Material Loss}

The median rate of material loss from the taper surfaces of the MOM and MOP groups was $0.81 \mathrm{~mm}^{3} /$ year $(0.01-3.45)$ and $0.03 \mathrm{~mm}^{3} /$ year $(0-1.07)$ respectively, Figure 5. The total volume of material lost from both groups was $6.13 \mathrm{~mm}^{3}(0.09-23.42)$ and $0.15 \mathrm{~mm}^{3}(0-3.80)$; typical 
wear maps of the taper surfaces are presented in Figure 6. Of the single MOP hip that was revised for ARMD, the total taper material lost was $3.8 \mathrm{~mm}^{3}$ and the annual rate was 0.35 $\mathrm{mm}^{3} /$ year.

Linear regression was used to compare the taper wear rate between the two hip types, Table 4 . Due to the analysis on the log scale, the difference in outcome between groups is expressed as a ratio. This gives the ratio of wear rate in the MOP group relative to the wear rate in the MOM group. Corresponding confidence intervals are also reported, along with p-values indicating the significance of the results.

The results suggested that there was a significant difference between the two hip types in the analysis unadjusted for possible confounding variables. Wear rate was significantly lower in the MOP group, with wear rate values that were only 0.27 times as large (or $73 \%$ smaller) than those in the MOM group. The results were almost unchanged after adjusting for baseline factors found to vary between the two hip types.

\section{Detailed Visual Inspection}

Visual inspection of the bearing surfaces of the MOM hips revealed light scratching of all components. A clear wear scar was visible on 19 out of 30 hips; 9 of the metal liners had clear scars at their rims. 23 out of 30 of the metal liners were available for inspection of their backside surfaces. Virtually all components were found to have minimal damage at this junction, limited to light scratching and mild discolouration. One case had evidence of mild corrosion with some pitting of the surface.

Inspection of the MOP bearing surfaces showed that all components had evidence of light scratching. 3 out of 22 of the polyethylene liners had evidence of mild deformation or delamination of the surfaces near the rims. Examination of the backside of the polyethylene 
liners showed light scratching at the point of engagement between the shell and liner however there was no considerable damage at this junction.

\section{Discussion}

\section{Summary}

We sought to better understand the function of the head-stem taper junction in patients by studying retrieved hips together with clinical and imaging data. Whilst there has been a recent increase in reports on this subject for MOM hips, there have been very few reports for MOP hips and our study is one of the first to compare the effect of bearing type on head-stem taper wear for one hip system. We used multi-variate statistical analysis of seven variables from 52 hips (30 MOM and $22 \mathrm{MOP})$ and found that the amount of corrosion and volumes of material loss at the tapers of MOP hips was significantly less $(\mathrm{p}<0.001)$ than in the MOM hips.

\section{Clinical Relevance}

Our study has immediate clinical relevance to the estimated 400,000 patients who have the Pinnacle system implanted with metal-on-polyethylene bearings. These patients can be reassured that wear of the head-stem taper junction is unlikely to cause them clinically significant problems. This is a pertinent discovery because of recent reports that several designs of metal on polyethylene hips have caused catastrophic wear at this junction $[7,8,15]$.

\section{Quantification of Material Loss from Taper Junction}

The methods for analysing material lost at this junction have improved dramatically in recent years so it has only been recently possible to estimate a clinically relevant level of taper material loss. 
The rate of material loss at the taper junction of the MOM group was $0.81 \mathrm{~mm}^{3} / \mathrm{year}$, which is approximately 10 -fold greater than those previously reported as being clinically insignificant $\left(0.084 \mathrm{~mm}^{3} /\right.$ year$)[12]$. The rate of material loss was 3 times greater from the bearing surfaces when compared to the taper surfaces and because Cobalt-Chromium is the main constituent of the material loss from both areas, it is difficult to establish the clinical effect of material from the taper surfaces of MOM hips.

In contrast, the rate of material loss from the taper surfaces of the MOP group was similar to that found in hips revised for reasons other than ARMD [12]. This makes sense because only one of the MOP patients were revised due to ARMD. It is of note that the rate of taper material loss of this patient was over 11-fold greater than the median rate of all the MOP hips however still less than half that of the MOM group.

We found no difference in the severity of corrosion of material loss between using a Corail or Summit stem in both groups. This also makes sense given the similarities in trunnion geometry and material between the two stems.

\section{Strengths of our study}

Our study is the first to compare the extent and severity of corrosion and material loss at the taper junction surface of MOM and MOP bearing hips of a single design. We consecutively recruited a relatively large number of MOP hips. We used highly accurate measurement methods and then used multivariate statistical analysis to control for differences identified between the two groups and found that the MOP group has significantly less taper corrosion and material loss than in the MOM group. 


\section{Limitations}

Our study has two main limitations. First, our sample was only 30 and 22 in each group. However, a group of $22 \mathrm{MOP}$ retrievals with comparative $30 \mathrm{MOM}$ retrievals of the same design makes our study one of the largest retrieval studies of its kind. Secondly, the recruitment process led to potential confounding variables between the two groups, with dissimilar means for time to revision, head diameter and reasons for revision. This is understandable given that the failure rate of MOP hips is low and often explainable, such as from infection or malposition, so surgeons do not keep the retrievals for analysis. In contrast, the generally higher revision rates of MOM hips are well documented and more likely to be related to ARMD, due to metal debris released from the hard-on-hard bearing.

There was also a difference in the head size between the groups. All MOM hips were $36 \mathrm{~mm}$ whilst $45 \%$ of heads in the MOP group had smaller diameters. Head diameter is positively correlated with frictional torque at the bearing [16] which is transmitted to the head-stem taper junction. However, the difference in head size in the current study may be mitigated by the lower frictional torque found with bearings that have fluid-film lubrication, such as MOM.

It is also difficult to quantify patient activity levels and relate these to retrieval findings. However, age can be a surrogate for activity and this was similar in both groups. We note however that the majority of the reasons for revision of the MOP group are likely to have been associated with considerable pain. Coupled with the shorter time to revision of this group, it may be that patients with MOP hips were less active over a shorter period of time.

\section{Mechanism of Material Loss from the Taper Surfaces of MOM hips}

Unlike the bearing surfaces, the head-stem taper junction is not expected to wear or corrode because there should be no movement or fluid ingress at the junction. Numerous studies suggest the following sequence of events: large head diameter; increased frictional torque; increased 
turning force transferred from the bearing to the head-stem junction; increased micro-motion at the taper junction; resulting in greater mechanically-assisted corrosion $[13,16]$. Our study suggests that the presence of metal debris from the bearing surface is also probably an important factor for generating clinically significant levels of material loss form the taper junction because $55 \%$ of the MOP hips had a bearing diameter of $36 \mathrm{~mm}$ (all MOM hips had a bearing diameter of $36 \mathrm{~mm}$ ) and yet had minimal material loss from the taper junction. We suggest a mechanism whereby there is an electrochemical current transfer from the MOM bearing to the taper junction, leading to corrosion and material loss. We also speculate that synovial fluid containing metal ions released from the metal bearing surfaces enter the junction between the stem and head and strengthen the electrochemical galvanic corrosion process taking place.

\section{Conclusion}

The rate of material loss from the head-stem taper junctions of Pinnacle MOP hips is negligible, clinically insignificant and 27-fold less than Pinnacle MOM hips. 


\section{References}

[1] National Joint Registry for England and Wales (NJR) 13th annual report, 2016, www.njrcentre.org.uk

[2] Goldberg JR, Gilbert JL, Jacobs JJ, Bauer TW, Paprosky W and Leurgans S. 2002. A multicentre retrieval study of the taper interfaces of modular hip prostheses. Clin Orthop, 401: 149-161.

[3] Hothi HS, Matthies AK, Berber R, Whittaker RK et al. 2014. The reliability of a scoring system for corrosion and fretting, and its relationship to material loss of tapered, modular junctions of retrieved hip implant. The Journal of Arthrplasty, 29(6): 1313-1317.

[4] Cross MB, Esposito C, Sokolova A, Jenabzadeh R, Molloy D, Munir S, Zicat B, Walter WK and Walter WL. 2013. Fretting and corrosion changes in modular total hip arthroplasty. Bone Joint J, 95-B (SUPP 15) 127.

[5] Higgs GB, Hanzlik JA, MacDonald DW, Gilbert JL, Rimna CM, Kurtz SM. 2013. Is increased modularity associated with increased fretting and corrosion damage in metal-on-metal total hip arthroplasty devices? A retrieval study. The Journal of Arthroplasty, 28(1): 2-6.

[6] Junnila M, Kostensalo I, Virolainen P, Remes V, Matilainen M, Vahlberg T, Pulkkinen P, Eskelinen A, Itala A, Makela K. 2014. Hip resurfacing arthroplasty versus large-diameter head metal-on-metal total hip arthroplasty: comparison of three designs from the Finnish Arthroplasty Register. Scand J Surf, 103(1): 54-59

[7] Cooper JH, Della Valle CJ, Berger RA, Tetreault M, Paprosky WG, Sporer SM, Jacobs JJ. 2012. Corrosion at the head-neck taper as a cause for adverse local tissue reactions after total hip arthroplasty. J Bone Joint Surg Am, 94(18): 1655-1661 
[8] Eltit F, Assiri A, Garbuz D, Duncan C, Masri B, Greidanus N, Bell R, Sharma M, Cox M, Wang R. 2017. Adverse reactions to metal on polyethylene implants: Highly destructive lesions related to elevated concentration of cobalt and chromium in synovial fluid. J Biomed Mater Res A, Epub ahead of print, March $7^{\text {th }}$

[9] Whittaker RK, Hothi HS, Eskelinen A, Blunn GW, Skinner JA, Hart AJ. 2016. The variation in taper surface roughness for a single design effects the wear rate in total hip arthroplasty. JOR, epub ahead of print, October $5^{\text {th }}$

[10] Hothi HS, Whittaker RK, Meswania JM, Blunn GW, Skinner JA, Hart AJ. 2015. Influence of stem type on material loss at the metal-on-metal pinnacle taper junction. IMechE H, 229(1): 91-97

[11] Hothi HS, Eskelinen AP, Berber R, Lainiala OS, Moilanen TPS, Skinner JA, Hart AJ. 2017. Factors associated with trunnionosis in the metal-on-metal Pinnacle hip. J Arthroplasty, 32(1): 286-290

[12] Hothi HS, Kendoff D, Lausmann C, Henckel J, Gehrke T, Skinner J, Hart A. 2017. Clinically insignificant trunnionosis in large-diameter metal-on-polyethylene total hip arthroplasty. Bone Joint Res, 6(1): 52-56.

[13] Matthies AK, Racasan R, Bills P, Blunt L, Cro S, Panagiotidou A, Blunn G, Skinner J and Hart AJ. 2013. Material loss at the taper junction of retrieved large head metal-on-metal total hip replacements. J Orthop Res, 31(11): 1677-1685.

[14] Hothi HS, Berber R, Whittaker RK, Bills PJ, Skinner JA, Hart AJ. 2015. Detailed inspection of metal hips. Hip Int, 25(3): 227-231 
[15] No authors listed. 2016. Class 2 device recall Stryker LFIT anatomic V40 femoral head. Available at https://www.accessdata.fda.gov/scripts/cdrh/cfdocs/cfRes/res.cfm?ID=149782 [last accessed 04/04/2017]

[16] Dyrkacz RMR, Brandt J, Ojo OA, Turgeon TR and Wyss UP. 2013. The influence of head size on corrosion and fretting behaviour at the head-neck interface of artificial hip joints. J Arthroplasty, 28: 1036-1040. 


\begin{tabular}{|l|l|c|c|c|}
\hline Variable & Category & $\begin{array}{c}\text { MOM } \\
(\mathrm{n}=30)\end{array}$ & $\begin{array}{c}\text { MOP } \\
(\mathrm{n}=22)\end{array}$ & P-value \\
\hline Head size (mm) & $\begin{array}{l}28 / 32 \\
36\end{array}$ & $\begin{array}{c}0(0 \%) \\
30(100 \%)\end{array}$ & $\begin{array}{l}10(45 \%) \\
12(55 \%)\end{array}$ & $<\mathbf{0 . 0 0 1}$ \\
Gender & $\begin{array}{l}\text { Female } \\
\text { Male }\end{array}$ & $\begin{array}{l}13(43 \%) \\
17(57 \%)\end{array}$ & $\begin{array}{l}12(55 \%) \\
10(45 \%)\end{array}$ & 0.58 \\
Age (years) & - & $62.9 \pm 6.1$ & $62.2 \pm 12.5$ & 0.78 \\
Time revisions & - & $91(18)$ & $54(49)$ & $<\mathbf{0 . 0 0 1}$ \\
(months) & - & $45.8(6.7)$ & $45.3(6.4)$ & 0.76 \\
Inclination & & $45.6(5.9)$ & $44.5(7.0)$ & 0.51 \\
Horizontal offset (mm) & - & $78.1(9.5)$ & $78.4(10.9)$ & 0.91 \\
Vertical offset (mm) & - & & & \\
\hline
\end{tabular}

Table 1: Summary of the differences in the baseline characteristics between the two groups. The figures are the number and percentage in each group for the categorical variables, or the mean and standard deviation for the continuous measures.

\begin{tabular}{|c|c|c|}
\hline Goldberg score & $\begin{array}{c}\text { MOM } \\
\text { Number }(\%)\end{array}$ & $\begin{array}{c}\text { MOP } \\
\text { Number }(\%)\end{array}$ \\
\hline 1 & $0(0 \%)$ & $10(45 \%)$ \\
2 & $4(13 \%)$ & $7(32 \%)$ \\
3 & $9(30 \%)$ & $4(18 \%)$ \\
4 & $17(57 \%)$ & $1(5 \%)$ \\
\hline
\end{tabular}

Table 2: Summary of taper corrosion scores for the two groups

\begin{tabular}{|l|c|r|}
\hline Adjustments & Odds Ratio $^{(*)}(95 \% \mathrm{CI})$ & \multicolumn{1}{|c|}{ P-value } \\
\hline None & $0.04(0.01,0.14)$ & $<\mathbf{0 . 0 0 1}$ \\
Head size, time revision & $0.05(0.01,0.27)$ & $\mathbf{0 . 0 0 1}$ \\
\hline
\end{tabular}

Table 3: $(*)$ Odds ratios given as values for MOP group relative to MOM group

\begin{tabular}{|l|c|c|}
\hline Adjustments & Ratio $^{(*)}(95 \% \mathrm{CI})$ & P-value \\
\hline None & $0.26(0.15,0.44)$ & $<\mathbf{0 . 0 0 1}$ \\
Head size, time revision & $0.27(0.12,0.60)$ & $\mathbf{0 . 0 0 2}$ \\
\hline
\end{tabular}

Table 4: (*) Ratios given as values for the MOP group relative to MOM group 


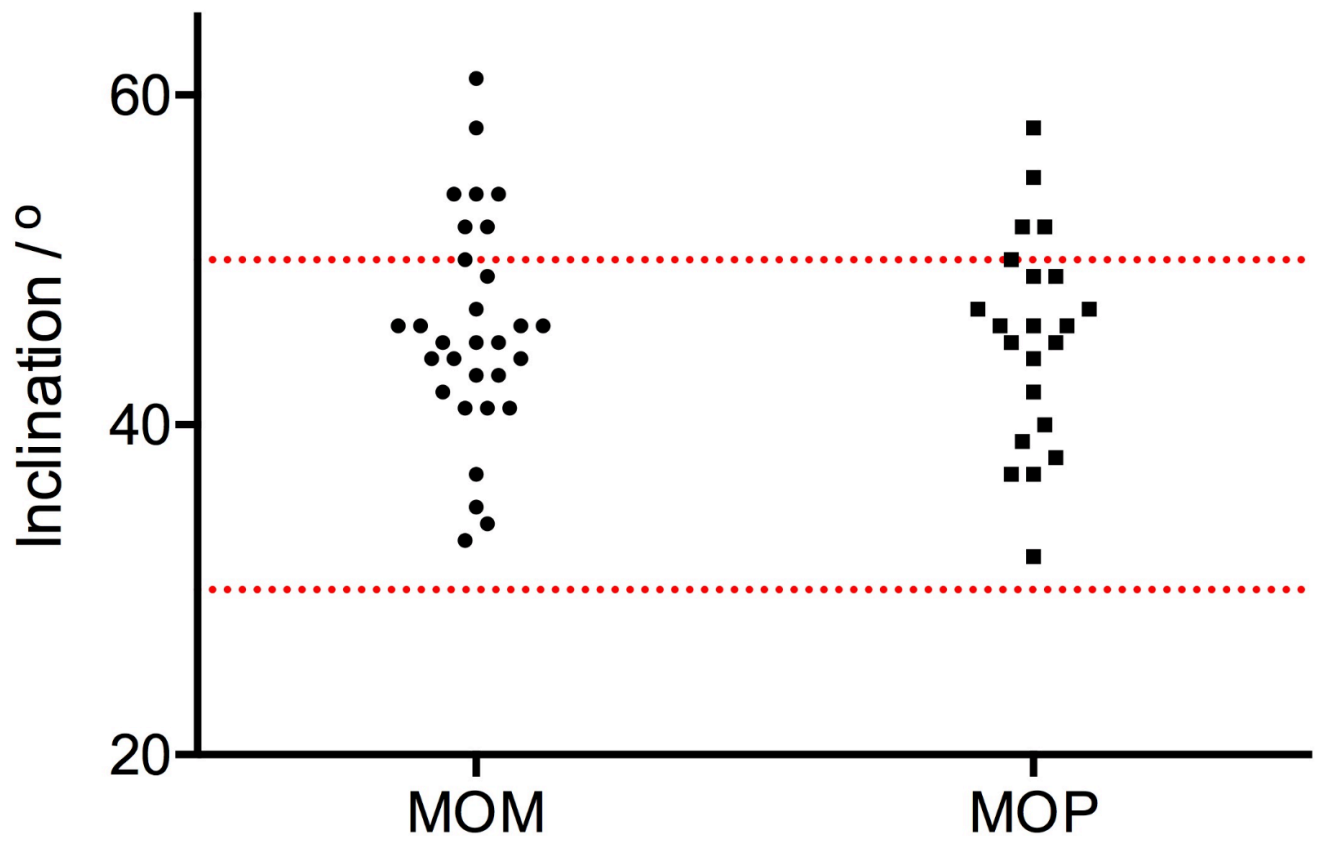

Figure 1: Distribution of the measurements of cup inclination of the implants in the two groups. The vertical lines represent the upper $\left(50^{\circ}\right)$ and lower $\left(30^{\circ}\right)$ limits of the safe zone defined by Lewinnek.

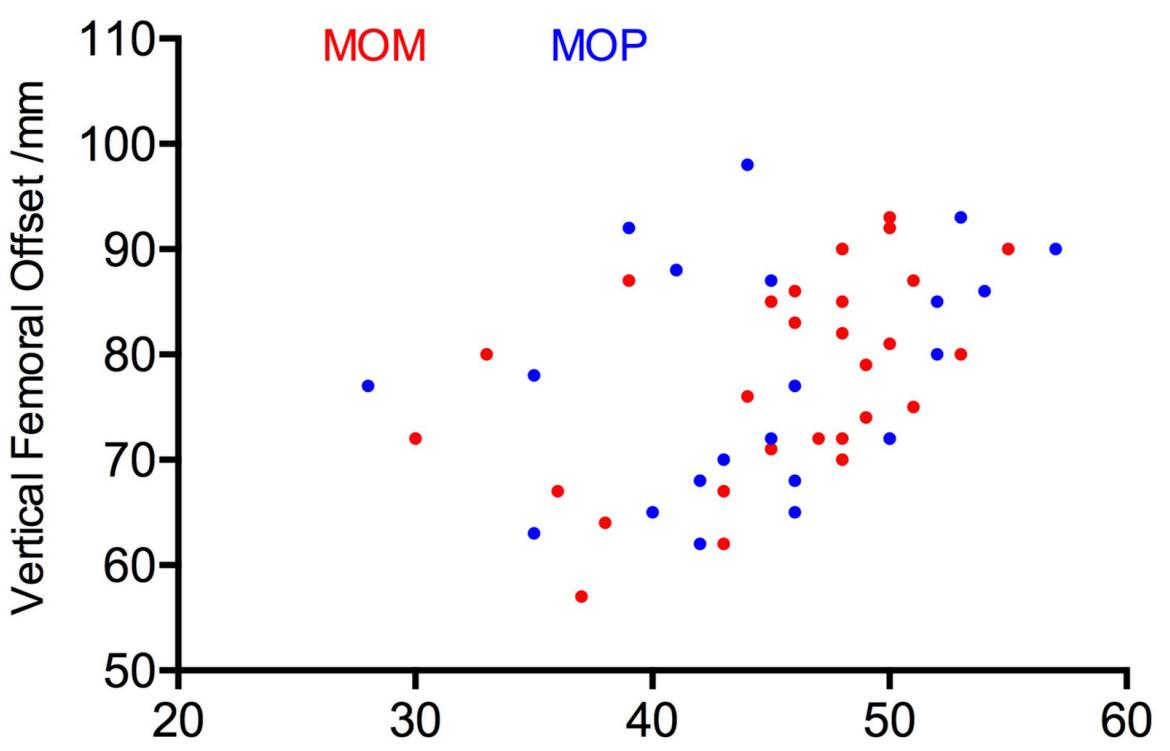

Figure 2: Distribution of the measurements of horizontal and vertical femoral offset of the two groups. 


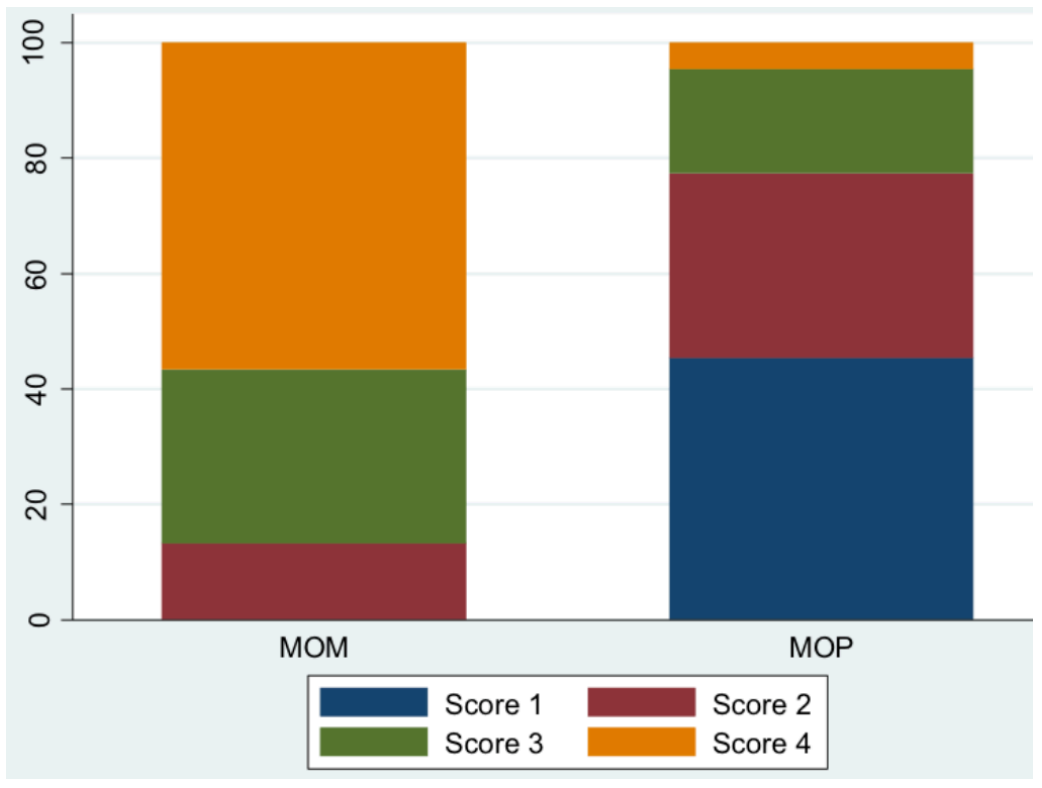

Figure 3: Distribution of the Goldberg corrosion scores for the taper surfaces of the two bearing types

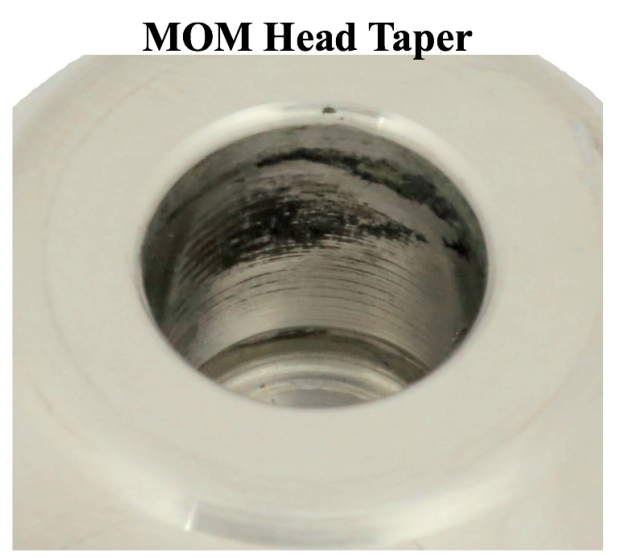

Corrosion score $=4$
non-MOM Head Taper

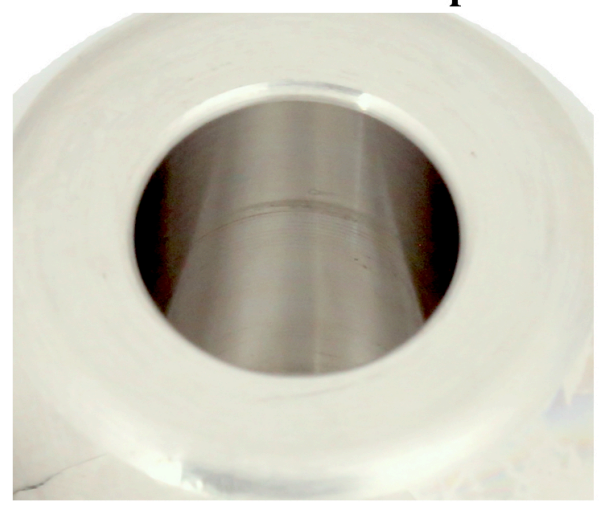

Corrosion score $=2$

Figure 4: Examples of taper damage observed in the MOM (left) and MOP (right) groups 


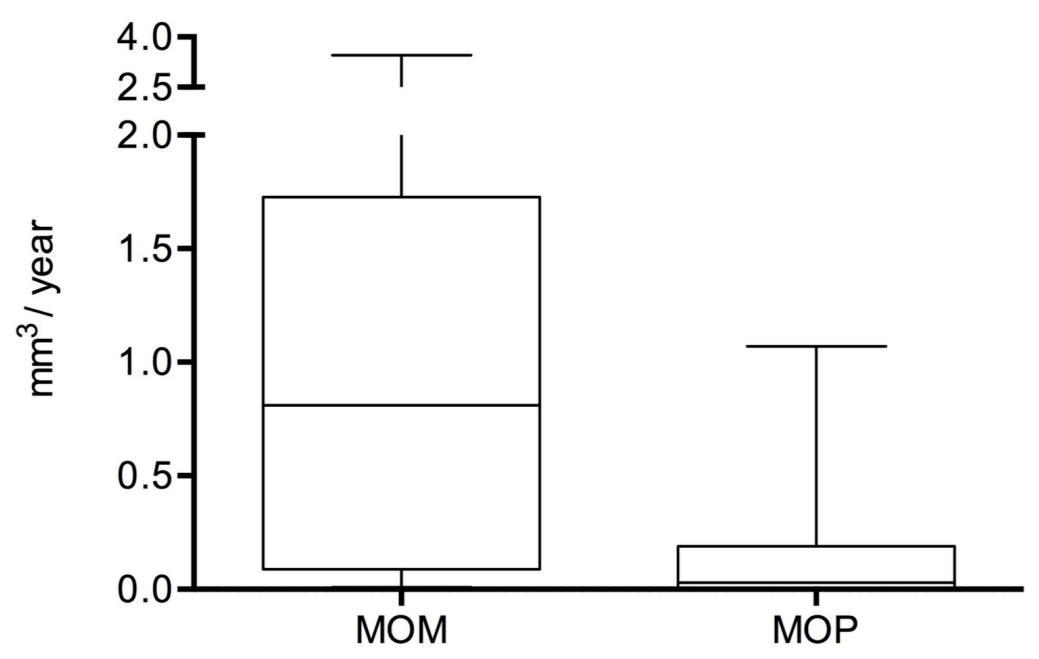

Figure 5: Box plots of the rate of volumetric material loss from the taper surfaces of the two bearing types

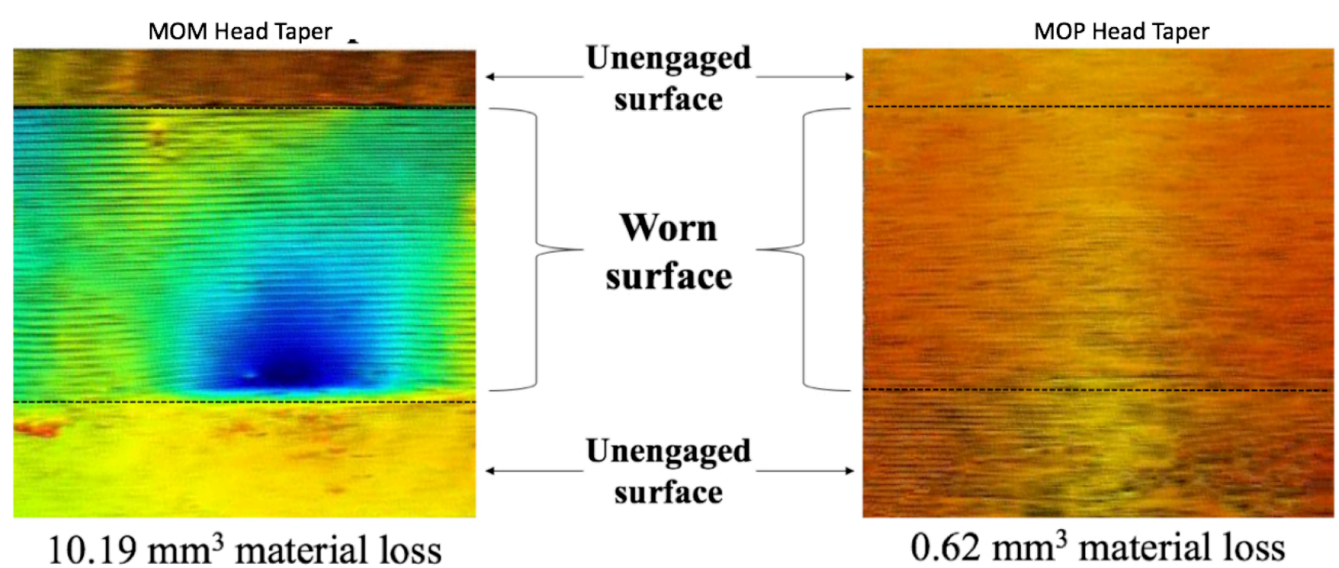

Figure 6: Wear maps generated from the roundness measuring machine showing a typical scan from the surface of a MOM head taper (left) and MOP taper (right). Both heads in this example were paired with a Corail stem. The worn regions are representative of the areas of engagement between stem trunnion and head taper

\section{Acknowledgments}

We thank Anna Di Laura for support in retrieval analysis, Paul Bassett for all statistical analysis and Daniel Kendoff, Mustafa Citak and Christian Lausmann for support in recruiting implants to this study. 